\title{
Investigation of the Electromagnetic Radiation Emitted by Sub-GeV Electrons in a Bent Crystal
}

\author{
L. Bandiera, E. Bagli, G. Germogli, V. Guidi, and A. Mazzolari \\ INFN Sezione di Ferrara and Dipartimento di Fisica e Scienze della Terra, \\ Università degli Studi di Ferrara Via Saragat 1, 44122 Ferrara, Italy \\ H. Backe and W. Lauth \\ Institut für Kernphysik der Universität Mainz, Fachbereich Physik, \\ Mathematik und Informatik, D-55099 Mainz, Germany \\ A. Berra, D. Lietti, and M. Prest \\ Università degli Studi dell'Insubria, Via Valleggio 11, 22100 Como, Italy \\ and INFN Sezione di Milano Bicocca, Piazza della Scienza 3, 20126 Milano, Italy \\ D. De Salvador \\ INFN Laboratori Nazionali di Legnaro, viale dell'Università 2, 35020 Legnaro, Italy \\ and Dipartimento di Fisica, Università Di Padova, Via Marzolo 8, 35131 Padova, Italy \\ E. Vallazza \\ INFN Sezione di Trieste, Via Valerio 2, 34127 Trieste, Italy \\ V. Tikhomirov \\ Research Institute for Nuclear Problems, Belarusian State University, 220030 Minsk, Belarus
}

(Received 17 January 2015; published 9 July 2015)

\begin{abstract}
The radiation emitted by $855 \mathrm{MeV}$ electrons via planar channeling and volume reflection in a 30.5- $\mu \mathrm{m}$-thick bent $\mathrm{Si}$ crystal has been investigated at the MAMI (Mainzer Mikrotron) accelerator. The spectral intensity was much more intense than for an equivalent amorphous material, and peaked in the $\mathrm{MeV}$ range in the case of channeling radiation. Differently from a straight crystal, also for an incidence angle larger than the Lindhard angle, the spectral intensity remains nearly as high as for channeling. This is due to volume reflection, for which the intensity remains high at a large incidence angle over the whole angular acceptance, which is equal to the bending angle of the crystal. Monte Carlo simulations demonstrated that incoherent scattering significantly influences both the radiation spectrum and intensity, either for channeling or volume reflection. In the latter case, it has been shown that incoherent scattering increases the radiation intensity due to the contribution of volume-captured particles. As a consequence, the experimental spectrum becomes a mixture of channeling and pure volume reflection radiations. These results allow a better understanding of the radiation emitted by electrons subjected to coherent interactions in bent crystals within a still-unexplored energy range, which is relevant for possible applications for innovative and compact $\mathrm{x}$-ray or $\gamma$-ray sources.
\end{abstract}

DOI: 10.1103/PhysRevLett.115.025504

PACS numbers: 61.85.+p, 29.27.-a

The electromagnetic radiation emitted in the interaction of electron or positron beams with crystals has been investigated for years and in some cases exploited for the production of intense electromagnetic radiation [1-4]. The main feature of this kind of radiation [5-7] is the higher intensity of hard photoproduction compared to the case of bremsstrahlung in an amorphous medium, which is described by the Bethe and Heitler formulation (BH) [8]. The enhancement of the bremsstrahlung spectrum of electrons or positrons crossing the periodic structure of a crystal, which appeared for the first time in the works of Ferretti [9], Ter-Mikaelian [10], Dyson and Uberall [11], leads to the possibility of exploiting this type of radiation as a source of intense $\mathrm{x}$ rays or $\gamma$ rays, far harder than the photon energies achievable in magnetic undulators at the same primary electron beam energy. Such an effect took the name coherent bremsstrahlung (CB) and was experimentally proven true by Diambrini-Palazzi et al. at Frascati in 1960. In 1964, Lindhard introduced the concept of coherent scattering of charged particles by the atoms of a crystal when the particle trajectory is nearly aligned with a crystal axis or plane. In such low-angle approximation, correlations between successive collisions of a particle with the atoms in the same row or plane occur [12] and one can replace the potentials of separate atoms with an average continuous potential of the crystal atomic string or plane. 
The atomic string or plane steers a particle away from or toward the lattice atoms, depending on the particle's charge sign. If the incident angle with respect to the crystallographic axes or planes is smaller than $\theta_{L}=\sqrt{2 U_{0} / p v}$, charged particles can be confined inside the axial or planar potential well, $U_{0}$ being the potential well depth, $p$ and $v$ the particle momentum and velocity, respectively. This effect is called channeling and, even though it was discovered by computer simulations in 1963 [13], only the introduction of the continuous potential by Lindhard allowed a simple description and understanding of such a phenomenon. Channeled particles oscillate during their motion inside the planar or axial potential well, leading to a peculiar process of radiation emission by electrons or positrons, thereby called channeling radiation (CR) [14].

Even though CB and CR in straight crystals have been extensively investigated, new opportunities for intense production of electromagnetic radiation are offered by the usage of bent crystals. In particular, the radiation accompanying volume reflection (VR) in curved crystals is promising for relevant applications, spanning from a $\gamma$-ray or a positron source to a crystal-based collimation in future electron or positron colliders [15-17]. VR consists of the deflection of over-barrier particles to a direction opposite that of the crystal bending by an angle of the order of $\theta_{L}$ [18]. Because the particles that suffer VR are found in over-barrier states, they cannot be affected by dechanneling, leading to higher deflection efficiencies than for the channeling case, for either positive [19,20] or negative [21] particles. The lower dependence of the radiation accompanying VR on the particle charge sign makes this radiation very attractive for applications in electron accelerators, which are much more common, less expensive, and often offer the best performance in terms of emittance than currently available positron accelerators. Another advantage of the radiation accompanying VR is the large and adjustable angular acceptance for its generation. Indeed, the angular acceptance is practically equal to the bending angle of the crystal and considerably exceeds those for CB and $\mathrm{CR}$ in a straight crystal [22]. This feature would open up new possibilities in application with the usage of relatively poor-emittance electron beams.

The few existing studies on VR radiation have been done with hundreds-GeV beams [16,22-25]. In those experiments, mm-long bent crystals were used, while, since the dechanneling length for $\mathrm{GeV}$ electrons is of the order of $20 \mu \mathrm{m}$ [26], bent crystals with a thickness comparable to such a length are needed to steer sub-GeV electron beams. Only very recently, such a possibility was demonstrated for either channeling or VR [27,28]. These results opened up a new challenge for electromagnetic radiation generation in bent crystals within the energy range accessible by many electron accelerators worldwide. In fact, there is some wealth of data concerning the radiation emitted by electrons in straight crystals in the sub-GeV or GeV energy ranges, while no experimental data on radiation in bent crystals exists in the literature - only theoretical expectations $[29,30]$.

In this Letter, we report on a study about the radiation generated by $855 \mathrm{MeV}$ electrons through coherent interaction with a bent crystal. The electron beam was accelerated at the Mainzer Mikrotron [31]. The study aims at investigating the influence of the curvature on the radiation emitted by electrons in a bent crystal and thereby the radiation accompanying VR within an energy range interesting for an $\mathrm{x}$-ray or $\gamma$-ray source.

The experimental setup of Ref. [26] was upgraded with a microstrip Si detector with a spatial resolution of $10 \mu \mathrm{m}$ [32] in order to reconstruct the beam profile after interaction with the crystal [27]. The photons emitted by the electrons inside the crystal were separated by the charged beam through a bending magnet and after $8.627 \mathrm{~m}$ arrived at a $25.4 \times 25.4 \mathrm{~cm}^{2} \mathrm{NaI}$ scintillator detector. An aperture of $40-\mathrm{mm}$ diameter in the lead shield surrounding the detector permitted the collection of a portion of the emitted photons, resulting in a collimator aperture of $4.63 \mathrm{mrad}$, i.e., equal to $\sim 7.8$ times the $1 / \gamma$ angle, for collection of most of the emitted photons. More details on the experimental setup can be found in Ref. [33]. Following the idea presented in Ref. [34], a Si crystal was bent through the quasimosaic effect [35] along its (111) planes. Crystal thickness along the beam was $(30.5 \pm 0.5) \mu \mathrm{m}$ and its bending radius was $33.5 \mathrm{~mm}$, approximately 23 times the critical radius for channeling at this energy $\left(R_{c}=1.5 \mathrm{~mm}\right)$ [27,36]. Beam divergence was about $30 \mu \mathrm{rad}$ of standard deviation along the direction transverse to the (111) planes, i.e., much less than $\theta_{L}$, which is about $220 \mu \mathrm{rad}$ at $855 \mathrm{MeV}$.

Before measuring the radiation spectra, the particle dynamics were investigated in order to ensure the proper experimental conditions for channeling and VR, while probing, at the same time, the steering capability of the crystal [27]. Figure 1(a) represents the distribution of the beam particles after the interaction with the crystal vs the crystal-to-beam orientation. The labels (1)-(6) on the figure highlight different regions of interest. In regions (1) and (6), the crystal is not aligned with the beam, so that neither channeling nor VR occur. Region (2) corresponds to planar channeling, while region (3) is populated by dechanneled particles. Dechanneling consists of the ejection of a particle from a channel, due to incoherent scattering suffered by the particle on crystal nuclei and electrons. After a dechanneling event, the transverse energy of a channeled electron becomes higher than the planar potential barrier, i.e., a particle passes from an under- to an overbarrier state. Incoherent scattering may also cause rechanneling of previously dechanneled particles [27]. Regions (4) and (5) correspond to VR and volume capture (VC) [37], respectively. Those phenomena manifest themselves because the crystal is oriented in such a way that, even in the case of an incidence angle larger than $\theta_{L}$, the 


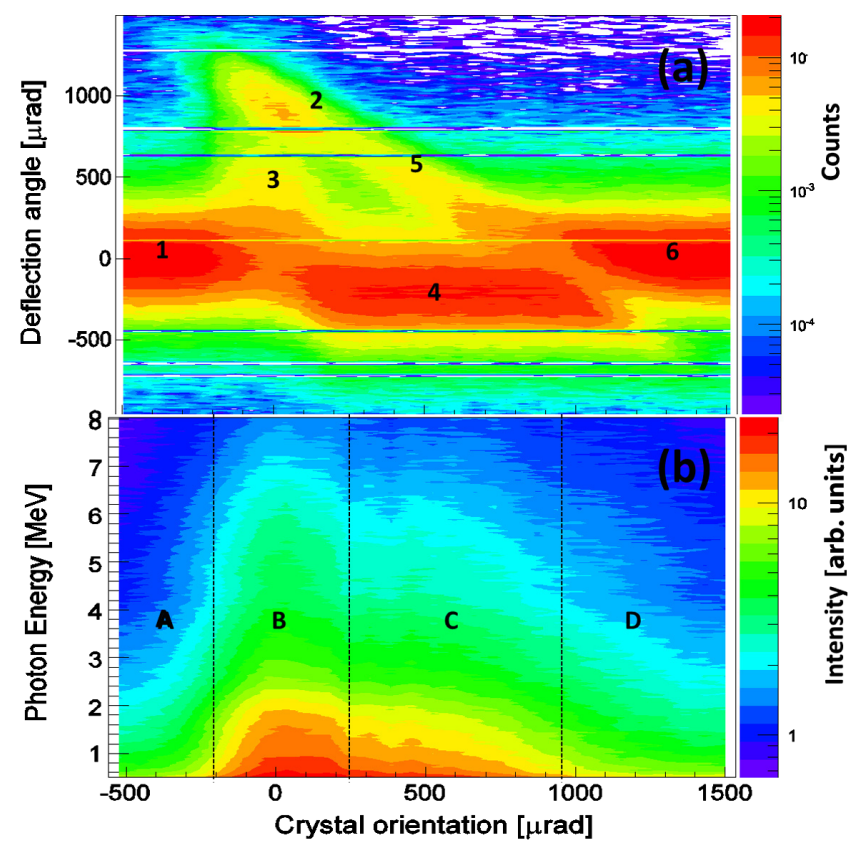

FIG. 1 (color online). (a) Experimental deflected beam distribution vs the incoming angle with respect to the bent (111) planes. Different regions of interest are labeled by different numbers: (1) and (6) crystal for the nonalignment case, (2) for channeling, (3) for dechanneling, (4) for VR, and (5) for VC. This is a reproduction of Fig. 2(a) in Ref. [27]. (b) Experimental radiation spectrum vs the incoming angle with respect to the bent (111) planes. Regions A and D correspond to the nonchanneling case, $\mathrm{B}$ is for channeling, and $\mathrm{C}$ for VR.

beam trajectory becomes tangent to the bent atomic planes in the crystal bulk. Under such conditions, most of the particles undergo VR [18], while a fraction of the beam is captured into the channeling regime (VC) due to incoherent scattering [19].

In order to measure the radiation spectrum, a calibration of the NaI detector was performed by using the natural radioactive isotopes ${ }^{40} \mathrm{~K}(1.461 \mathrm{MeV})$ and ${ }^{208} \mathrm{Tl}-{ }^{228} \mathrm{Th}$ $(2.6146 \mathrm{MeV})$. The energy scale has been selected in the region of interest for CR. The experimental radiation spectrum after interaction with the crystal as a function of the crystal-to-beam angle is shown in Fig. 1(b). Regions $\mathrm{A}$ and $\mathrm{D}$ correspond to the nonchanneling case, $\mathrm{B}$ is for channeling, and $\mathrm{C}$ for VR. Region $\mathrm{B}$ also includes the contribution of the radiation emitted by the particles that were dechanneled during their motions. Similarly, the contribution of volume-captured particles is also included in region $\mathrm{C}$. As expected, the radiation intensity is stronger for CR (B) than for the misaligned case (A and D). Moreover, the intensity in region $\mathrm{C}$ of Fig. 1(b) is still higher than for the misaligned case within the whole angular acceptance of VR, corresponding to $\sim 900 \mu \mathrm{rad}$. Figure 1(b) differs considerably if compared to the case of a straight crystal. Indeed, in the latter case the radiation intensity falls off very rapidly out of the channeling region,
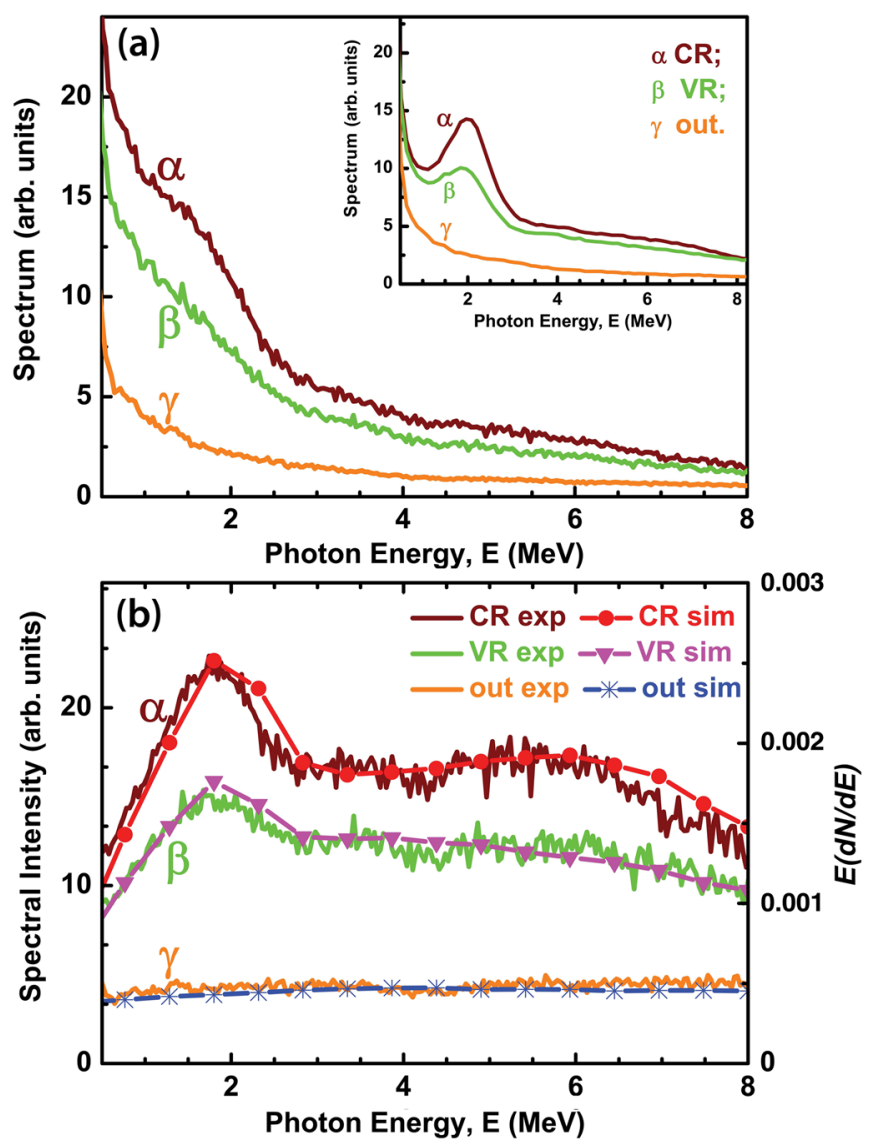

FIG. 2 (color online). (a) Experimental radiation spectra (solid lines) for the cases of channeling $\left(\theta_{\text {in }}=0 \mu \mathrm{rad}, \alpha\right)$, VR $\left(\theta_{\text {in }}=493 \mu \mathrm{rad}, \beta\right)$, and far from (111) bent planes $\left(\theta_{\text {in }}=8726 \mu \mathrm{rad}, \gamma\right)$. Beam-off background has been subtracted. The experimental scale has been chosen from $0.5 \mathrm{MeV}$ to $8 \mathrm{MeV}$ to select the region of interest for CR. Inset of Fig. 2(a): experimental radiation spectra (solid lines) for the cases of channeling $(\alpha)$, VR $(\beta)$, and far from (111) bent planes $(\gamma)$, obtained with a smaller collimator aperture $(4 \mathrm{~mm}$ instead of $40 \mathrm{~mm}$ ) compared to Fig. 2(a). (b) Experimental (left scale) and simulated (right scale) radiation spectral intensities obtained by multiplying the experimental spectra in Fig. 2(a) by the photon energy $E$ for the three cases. The photon emission intensity for one electron, $E(d N / d E)$, has been obtained averaging over 1,000 trajectories.

which is as large as $2 \theta_{L}=440 \mu \mathrm{rad}$, passing from CR to $\mathrm{CB}$ radiation.

Figure 2(a) shows the experimental spectra for the radiation emitted as the crystal was oriented in channeling ( $\alpha$ curve), in the middle of VR region ( $\beta$ curve), and far from the alignment condition with bent planes ( $\gamma$ curve, recorded at an angle of $\theta_{i n}=8726 \mu \mathrm{rad}$ from channeling). The experimental scale has been chosen from $0.5 \mathrm{MeV}$ to $8 \mathrm{MeV}$ to select the region of interest for CR. The depicted spectra are unprocessed, i.e., the deconvolution from the detector response function has not been performed. Nevertheless, the effect of the finite resolution would 
influence mostly the lower part of the spectra $(<0.5 \mathrm{MeV})$, far from the $\mathrm{CR}$ region [26]. The intensities for both $\mathrm{CR}(\alpha)$ and the radiation accompanying VR $(\beta)$ are stronger than for the case far from channeling $(\gamma)$. The experimental spectra represent the total radiation collected by the $\mathrm{NaI}$ detector, which is proportional to the number of photons by unit of time and energy. For the sake of better comparison with the spectra usually displayed in the literature, Fig. 2(b) highlights the spectral intensities obtained by multiplying the experimental spectra in Fig. 2(a) by the photon energy $E$. The spectral intensity of CR peaks for photon energies close to $1.8 \mathrm{MeV}$, which also appears in the case of VR, though less intense. Since many applications in nuclear physics or medicine do require nearly monochromatic $\mathrm{MeV}$ photon beams [38,39], we repeated the measurements for $\mathrm{CR}$ and radiation accompanying VR with a smaller collimator aperture (4 $\mathrm{mm}$ instead of $40 \mathrm{~mm}$ ) to reject the contribution of the soft radiation emitted at large angles. The experimental results show the possibility to pass from the total unpeaked spectra of Fig. 2(a) to the peaked ones displayed in the inset.

For the interpretation of experimentally recorded patterns, we performed Monte Carlo simulations with the code DYNECHARM++ [40] and its extension, including RADCHARM++ [30] for radiation computation. The DYNECHARM++ code takes into account the real particle trajectories, and thereby the contribution of incoherent scattering, too. The algorithm to compute the radiation emission probability implemented in RADCHARM++ is based on direct integration of the general formula of Baier and Katkov for the electromagnetic radiation generated by charged particles in an external field [41].

Displayed in Fig. 2 are the simulated spectral intensities $E(d N / d E)$ vs $E$, where $d N / d E$ is the photon emission probability for each electron, for channeling (circles), VR (down triangles), and misaligned cases (stars). Figure 2 shows a good agreement with the experiments in both shape and amplitude.

In Ref. [27], it was shown that incoherent scattering plays a crucial role in the electron dynamics under coherent interaction with the bent $30.5 \mu \mathrm{m}$ Si crystal, highlighting the contribution of dechanneling or rechanneling to the deflection efficiency. Figure 3 shows the simulated spectral intensities for CR (circles and squares) and VR (down and up triangles) by taking into account (circles and down triangles) or not taking into account (squares and up triangles) the incoherent scattering. For comparison, the simulated Bethe-Heitler value is also shown (diamonds). As expected, CR is suppressed by the contribution of incoherent scattering [42]. Indeed, since CR is generated by the oscillatory motion of particles inside the planar potential well, the scattering events that cause particle dechanneling lead to a reduction of CR intensity. On the contrary, when the crystal is oriented in VR condition, the incoherent scattering enhances the emitted radiation. This observation

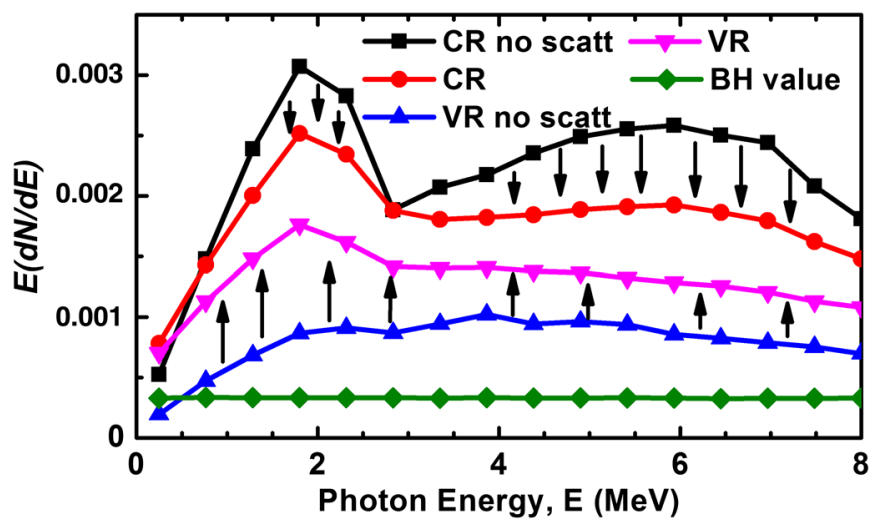

FIG. 3 (color online). Simulated spectral intensity $E(d N / d E)$ vs photon energy $E$ for channeling (circles and squares) and VR (down and up triangles) by taking (circles and down triangles) or not taking (squares and up triangles) into account the incoherent scattering. For comparison, the simulated Bethe-Heitler value is also shown (diamonds). The arrows directed downward underline the intensity decrease for CR caused by the incoherent scattering. The arrows directed upward underline the intensity increase, due to incoherent scattering, for the radiation accompanying VR.

is ascribed to the contribution of volume-captured particles. More precisely, after capture into the channel, such particles undergo dynamics typical of channeled particles, resulting in similar radiation processes as for CR. For a better understanding, we investigated through simulations the dynamics of those particles that contributed to the spectral intensity in Fig. 3 down triangles. These particles impinged onto the crystal at $493 \mu \mathrm{rad}$ from bent (111) planes, thereby they are either reflected or captured as shown in Fig. 1(a). It turned out that $68 \%$ of the initial population undergoes $\mathrm{VR}$, while the remaining $32 \%$ is captured into planar channeling as a result of $\mathrm{VC}$.

Figure 4 highlights the cases where all the particles were ideally volume reflected (curve 3 ) or volume captured (curve 1). The VR spectral intensity (curve 3 ) does not show any peak due to the change in the angle between particle trajectory and crystal planes as the particle approaches the reflection point (see trajectory 3 in the inset in Fig. 4) [22-24,41]. The VC spectral intensity (curve 1) is more intense than for VR and shows the typical spectrum for CR (Fig. 3, circles). Indeed, a captured electron is found in channeling condition under about half of its motion (see trajectory 1 in the inset in Fig. 4). Curve 2 represents the total contribution to radiation accompanying VR in real cases, in which the weighted VC (curve 1) and VR (curve 3) contributions are included.

The radiation accompanying VR is comparable in intensity to the radiation in a straight crystal; i.e., it is a sort of intermediate case between $\mathrm{CR}$ and $\mathrm{CB}$, and exhibits some important features. First of all, the radiation accompanying VR possesses an adjustable and broader angular acceptance than $\mathrm{CR}$ and $\mathrm{CB}$, which can be used for highintensity radiation generation with poor-emittance beams. 


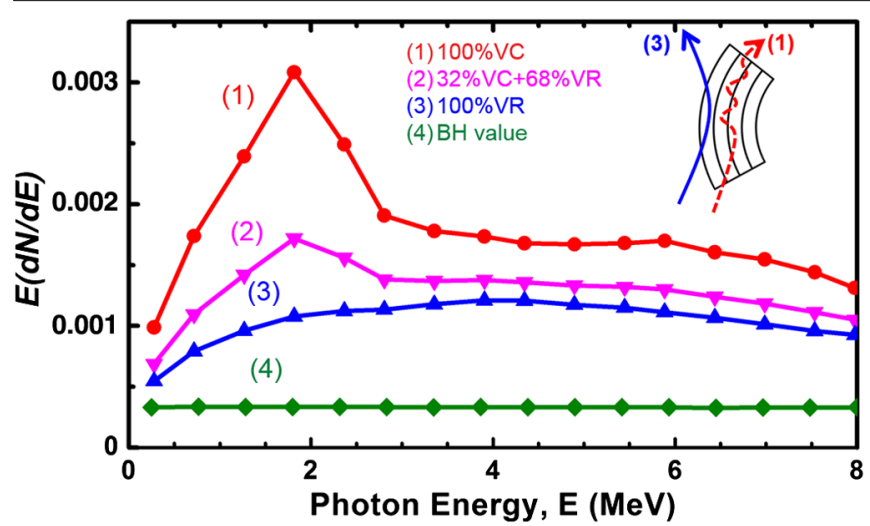

FIG. 4 (color online). Simulated spectral intensity $E(d N / d E)$ vs photon energy $E$ for (1) volume-captured particles, (3) volume-reflected particles, and (2) for the total radiation accompanying VR, including both the contributions (1) and (3). (4) The simulated Bethe-Heitler value is also shown. Inset: schematic representations of a captured (1) and a reflected (3) electron trajectory in a region close to the bent (111) planes.

Second, the steering efficiency of VR is less sensitive to crystalline defects compared to channeling [43], opening up a way to the usage of higher $Z$ materials such as $\mathrm{W}$, which usually cannot be grown with the same perfection as a $\mathrm{Si}$ crystal, while being more suitable for radiation generation $[43,44]$. Finally, the process of photon generation under VR conditions comes together with particle steering and, thereby, the combination of these two physical effects can be useful in practical applications. As an example, it was proposed to use VR and the accompanying radiation in a possible crystal-based collimation scheme for future electron-positron colliders [15]. The choice of VR instead of channeling can be preferable for some applications due to its higher deflection efficiency and wider angular acceptance.

In summary, the possibility to exploit bent crystals to generate hard and very intense electromagnetic radiation has been experimentally demonstrated and well reproduced by simulations. It has also been demonstrated that $\mathrm{CR}$ and the radiation accompanying VR are much more intense than for an amorphous material. In particular, it was shown how the radiation accompanying VR naturally combines the radiation emitted by pure volume-reflected particles with that emitted as a result of $\mathrm{VC}$. The contribution of $\mathrm{VC}$ radiation renders the radiation accompanying VR similar to that for CR in intensity, but its angular acceptance is wider than for channeling. The combination of these features provides interesting prospects for applications of the electromagnetic radiation generated in bent crystals for electron accelerators available worldwide.

We acknowledge the partial support of INFN under the CHANEL experiment and the European Commission under the CUTE Project No. (GA 269131). The research leading to these results has received funding from the European
Community's Seventh Framework Programme FP7/20072013 under Grant Agreement No. 227431. In particular, we acknowledge Dr. Gerald Klug and Dr. Eugen Eurich from Disco Europe (Munich, Germany) for their support in crystal manufacturing, Mr. Andrea Persiani and Mr. Claudio Manfredi of Perman (Loiano, Italy) for their support in crystal holder manufacturing, and Gilles Frequet from Fogale Nanotech for precise measurement of crystal thickness by means of a T-MAP IR interferometer.

*guidi@fe.infn.it

[1] J. D. Kellie et al., Nucl. Instrum. Methods Phys. Res., Sect. A 545, 164 (2005).

[2] D. Lohmann et al., Nucl. Instrum. Methods Phys. Res., Sect. A 343, 494 (1994).

[3] N. Shul'ga, Int. J. Mod. Phys. A 25, 9 (2010).

[4] T. Koenig and U. Oelfke, Phys. Med. Biol. 55, 1327 (2010).

[5] M. L. Ter-Mikaelian, High-Energy Electromagnetic Processes in Condensed Media (Wiley, New York, 1972).

[6] V. Baier, V. Katkov, and V. Strakhovenko, Electromagnetic Processes at High Energies in Oriented Single Crystals (World Scientific, Singapore, 1998).

[7] A. Akhiezer and N. Shulga, High-Energy Electrodynamics in Matter (Gordon and Breach, New York, 1996).

[8] H. Bethe and W. Heitler, Proc. R. Soc. A 146, 83 (1934).

[9] B. Ferretti, Nuovo Cimento 7, 118 (1950).

[10] M. Ter-Mikaelian, Zh. Exp. Teor. Fiz. 25, 296 (1953).

[11] H. Uberall, Phys. Rev. 103, 1055 (1956).

[12] J. Lindhard, Danske Vid. Selsk. Mat. Fys. Medd. 34, 14 (1965).

[13] M. T. Robinson and O.S. Oen, Appl. Phys. Lett. 2, 30 (1963).

[14] M. Kumakhov, Phys. Lett. A 57, 17 (1976).

[15] A. Seryi, Nucl. Instrum. Methods Phys. Res., Sect. A 623, 23 (2010).

[16] L. Bandiera, E. Bagli, V. Guidi, A. Mazzolari, A. Berra, D. Lietti, M. Prest, E. Vallazza, D. De Salvador, and V. Tikhomirov, Phys. Rev. Lett. 111, 255502 (2013).

[17] L. Bandiera, A. Mazzolari, E. Bagli, A. Berra, D. Lietti, D. De Salvador, V. Guidi, M. Prest, V. Tikhomirov, and E. Vallazza, J. Phys. Conf. Ser. 517, 012043 (2014).

[18] A. Taratin and S. Vorobiev, Phys. Lett. A 119, 425 (1987).

[19] W. Scandale et al., Phys. Rev. Lett. 98, 154801 (2007).

[20] E. Bagli et al., Eur. Phys. J. C 74, 1 (2014).

[21] W. Scandale et al., Phys. Lett. B 681, 233 (2009).

[22] Yu. A. Chesnokov, V. I. Kotov, V. A. Maisheev, and I. A. Yazynin, J. Instrum. 3, P02005 (2008).

[23] W. Scandale et al., Phys. Rev. A 79, 012903 (2009).

[24] D. Lietti et al., Nucl. Instrum. Methods Phys. Res., Sect. B 283, 84 (2012).

[25] L. Bandiera et al., Nucl. Instrum. Methods Phys. Res., Sect. B 309, 135 (2013).

[26] H. Backe, P. Kunz, W. Lauth, and A. Rueda, Nucl. Instrum. Methods Phys. Res., Sect. B 266, 3835 (2008).

[27] A. Mazzolari et al., Phys. Rev. Lett. 112, 135503 (2014).

[28] U. Wienands et al., Phys. Rev. Lett. 114, 074801 (2015).

[29] R. G. Polozkov, V. K. Ivanov, G. B. Sushko, A. V. Korol, and A. V. Solovâyov, Eur. Phys. J. D 68, 268 (2014). 
[30] L. Bandiera, E. Bagli, V. Guidi, and V. V. Tikhomirov, Nucl. Instrum. Methods Phys. Res., Sect. A 355, 44 (2015).

[31] A. Jankowiak, Eur. Phys. J. A 28, 149 (2006).

[32] D. Lietti, A. Berra, M. Prest, and E. Vallazza, Nucl. Instrum. Methods Phys. Res., Sect. A 729, 527 (2013).

[33] D. Lietti et al., Rev. Sci. Instrum. 86, 045102 (2015).

[34] V. Guidi, A. Mazzolari, D. De Salvador, and A. Carnera, J. Phys. D 42, 182005 (2009).

[35] Y. Ivanov, A. Petrunin, and V. Skorobogatov, J. Exp. Theor. Phys. Lett. 81, 99 (2005).

[36] G. Germogli, A. Mazzolari, L. Bandiera, E. Bagli, and V. Guidi, Nucl. Instrum. Methods Phys. Res., Sect. B 355, 81 (2015).

[37] Yu. A. Chesnokov, N. A. Galyaev, V. I. Kotov, S. V. Tsarik, and V. N. Zapolsky, Nucl. Instrum. Methods Phys. Res., Sect. B 69, 247 (1992).
[38] H. R. Weller, M. W. Ahmed, H. Gao, W. Tornow, Y. K. Wu, M. Gai, and R. Miskimen, Prog. Part. Nucl. Phys. 62, 257 (2009).

[39] D. Habs, T. Tajima, and V. Zamfir, Nucl. Phys. News 21, 23 (2011).

[40] E. Bagli and V. Guidi, Nucl. Instrum. Methods Phys. Res., Sect. B 309, 124 (2013).

[41] V. Guidi, L. Bandiera, and V. Tikhomirov, Phys. Rev. A 86, 042903 (2012).

[42] O. Bogdanov and S. Dabagov, J. Phys. Conf. Ser. 357, 012029 (2012).

[43] E. Bagli et al., Phys. Rev. Lett. 110, 175502 (2013).

[44] C. Gary, R. Pantell, M. Özcan, M. Piestrup, and D. Boyers, J. Appl. Phys. 70, 2995 (1991). 